\title{
Utilization of parboiled rice polish based diet with supplementation of phytase and carbohydrase in growing ducklings
}

\author{
B. Naher ${ }^{1}$, M. Y. Miah', M. M. Roahman ${ }^{3}$ and M. A. Wahid ${ }^{1}$ \\ ${ }^{1}$ Department of Poultry Science, Bangladesh Agricultural University, Mymensingh-2202, Bangladesh, ${ }^{2}$ Department of \\ Dairy and Poultry Science, Sylhet Agricultural University, Sylhet- 3100, Bangladesh and ${ }^{3}$ Office of the Divisional \\ Commissioner, Dhaka, Bangladesh, Email: myoumsau2003@gmail.com
}

\begin{abstract}
A total of 60 day old straight run pekin ducklings were fed ad libitum on 6 diets viz, $\mathrm{D}_{1}-15 \%$ parboiled rice polish (PRP) based diet, $D_{2}-D_{1}+$ Mixed enzyme (Carbohydrase $120 \mathrm{ppm} / \mathrm{kg}+$ phytase $1000 \mathrm{FYT} / \mathrm{kg}$ ), $\mathrm{D}_{3^{-}}-25 \% \mathrm{parboiled}$ rice polish (PRP) based diet, $D_{4}-D_{3}+$ Mixed enzyme ( Carbohydrase $120 \mathrm{ppm} / \mathrm{kg}+$ phytase $1000 \mathrm{FYT} / \mathrm{kg}$ ), $\mathrm{D}_{5}-35 \%$ parboiled rice polish (PRP) based diet, $D_{6}-D_{5}+$ Mixed enzyme ( Carbohydrase $120 \mathrm{ppm} / \mathrm{kg}+$ phytase $1000 \mathrm{FYT} / \mathrm{kg}$ ) for 84 days. Diets were supplied two stages containing 22.5, 22.5, 22.8\% CP for starter and 16.4, 16.9, 17\% CP for finisher. Increasing PRP levels without enzyme decreased live weight, feed intake and feed conversion but increase profitability without affecting livability. Addition of mixed enzyme promoted growth, feed conversion significantly $(P<0.01)$, but did not alter livability. The rate of improvement in performance for enzyme supplementation increased with increasing level of dietary PRP. Therefore, it was concluded that it is possible to reduce costly grain by using PRP in duck ration and supplementation of phytase and carbohydrase in PRP diet may be beneficial to improve performance of ducks.
\end{abstract}

Keywords: Carbohydrase, Duckling, Phytase, Rice polish

\section{Introduction}

Bangladesh is a riverine country and $16488 \mathrm{Km}^{2}$ of its total land area are Haors, Baors, Canals, Ponds and low lying water reservoirs (Asian Livestock, 1978), most of them can be efficiently utilized for duck production. Duck do not compete with chicken for feed because they scavenge in low laying water lodged area that is not suitable for chicken scavenging.

In poultry production, feed alone accounts about $65-70 \%$ of the total cost of production (Banergee, 1992). The higher price and non-availability and low quality of feed ingredients are the major constrains of poultry farming in Bangladesh. It may be alleviated through exploration of potential cheaper locally available feedstuffs and by introducing improved methods for better utilization of poor quality feeds.

Poultry compete directly with human and other livestock for grains such as wheat, maize etc. The grain usually constitutes about $50 \%$ of total diet formulated for poultry. Maize and wheat are also less available as compared to their demand. Therefore, use of expensive grains hardly permits economic diet formulation for profitable poultry production.

Among grain by-products, rice polish is the most abundant in Bangladesh and is reasonably cheaper. It might be used alternative to grains. Polish constitutes about $10 \%$ of paddy and is available in large quantities in major rice growing areas of the world (Houston and Kohler, 1970).

Polish obtained from parboiled rice is called parboiled rice polish (PRP). PRP contains more nutrients and little amount of antinutrinational factors like free fatty acids, saponin, hemaglutinin and tannin than raw rice polish, except its phytin-phosphorus content (Padua and Juliano, 1974, Barber and Barber, 1980). PRP is Eshawaraiah et al. 1988 almost comparable with wheat in nutrient concentration. Eshawaraiah et al. 1988 reported that it contain $13 \%$ crude protein and $3250 \mathrm{kcal} / \mathrm{kg}$. Beside some merits of PRP, it has some demerits such as it decreases feed intake, growth rate and feed utilization; its phytase-phosphorus reduces the phosphorus and calcium availability. Its non-strach polysaccharides (NSP) such as cellulose xylose, arabinose and galactonicacid are not easily digested by poultry. These adverse effects of PRP could possibly be overcome by dietary supplementation of exogenous phytase and carbohydrase. Addition of exogenous phytase and carbohydrase have been reported to improve feed utilization in broiler on PRP diet ( Moshad 2001). 
The present work was therefore, undertaken with the following objectives:

i) To investigate the performance of ducks fed on PRP diet fortified with phytase and carbohydrases at different levels.

ii) To determine the economic feasibility of using phytase and carbohydrase enzyme on PRP based diet in meat type duck.

\section{Materials and Methods}

The experiment was conducted with 60 day-old Pekin duckling for a period of 12 weeks. The ducklings were randomly distributed to six dietary treatments having two replications of 5 birds in each. The lay out of the experiment is shown in Table 1. The experimental room was divided into 12 equal littered floor pens by using wire-net and wooden materials. There were 6 dietary treatments in the study in which one was rice polish based control diet and the other five were carbohydrase and phytase based diets. The birds were fed a duck starter diet from 1 day to 14 days of age and finisher diet from 22 to 84 days of age. The feed was supplied ad libitum as dry mash. Fresh, cool and clean drinking water was supplied all the times during the whole experimental period. The ingredients were selected on the basis of availability in the local market. Maize, rice polish, soybean meal based control diet was prepared (Table 1).

Table 1. Per cent of ingredient used in duck starter diets (0-2 weeks) and finish starter diets (3-12 weeks)

\begin{tabular}{|c|c|c|c|c|c|c|}
\hline \multirow[t]{3}{*}{ Ingredients } & \multicolumn{6}{|c|}{ Dietary parboiled rice polish $\left(\mathrm{PRP}^{\star}\right) \%$} \\
\hline & \multicolumn{3}{|c|}{ Starter } & \multicolumn{3}{|c|}{ Finisher } \\
\hline & 15 & 25 & 35 & 15 & 25 & 35 \\
\hline PRP & 15 & 25 & 35 & 15 & 25 & 35 \\
\hline Maize $(\mathrm{kg})$ & 49 & 40 & 29 & 63 & 52 & 45 \\
\hline Soy. Meal $(\mathrm{kg})$ & 25 & 24 & 25 & 12 & 10 & 10 \\
\hline Soy. oil $(\mathrm{kg})$ & - & - & 1 & - & - & 1 \\
\hline Till Oil Cake(kg) & 10 & 10 & 9 & 9 & 12 & 8 \\
\hline Meat \& bone meal & 0.5 & 0.5 & 0.5 & 0.5 & 0.5 & 0.5 \\
\hline Salt $(\mathrm{kg})$ & 0.5 & 0.5 & 0.5 & 0.5 & 0.5 & 0.5 \\
\hline Vit. Mineral ** & + & + & + & + & + & + \\
\hline \multicolumn{7}{|c|}{ Calculated composition } \\
\hline $\mathrm{M} \mathrm{E}(\mathrm{Kcal} / \mathrm{kg})$ & 2925 & 2908 & 2951 & 3059 & 3012 & 3102 \\
\hline СР\% & 22.5 & 22.5 & 22.8 & 16.9 & 17 & 16.4 \\
\hline $\mathrm{Ca} \%$ & 0.66 & 0.59 & 0.63 & 0.59 & 0.61 & 0.63 \\
\hline $\mathrm{P} \%$ & 0.46 & 0.4 & 0.41 & 0.37 & 0.42 & 0.4 \\
\hline Lysine \% & 1.07 & 1.0 & 1.0 & 0.72 & 0.73 & 0.6 \\
\hline Methionine \% & 0.4 & 0.4 & 0.4 & 0.3 & 0.3 & 0.35 \\
\hline
\end{tabular}

${ }^{*}$ PRP at levels of 15,25 and $35 \%$ constituted PRP based diets $D_{1}, D_{3}, D_{5}$ respectively, while $D_{2}, D_{4}, D_{6} c-$ constituted treatment diets containing mixed enzyme ( Carbohydrase $120 \mathrm{ppm} / \mathrm{kg}+$ phytase $1000 \mathrm{FYT} / \mathrm{kg}$ )

** Added vitamin-mineral premix @2.5g/kg

The birds were immunized against Duck Plague and Duck Cholera. During the experimental period, initial and weekly body weight, feed consumption, temperature and relative humidity, mortality etc were recorded. To evaluate different treatments weight gain, feed conversion ratio, survivability, production number, performance index, production cost, dressing yield records etc parameters were considered. Data collected and calculated for different parameters were subjected to analysis of variance (ANOVA) using a MSTAT statistical computer package programme according to the principles of Completely Randomized Design (CRD). Least significant differences (LSD) were performed to compare the mean values having significant difference between treatments. 


\section{Results and Discussion}

The results of feeding different levels of parboiled rice polish (PRP) with and without mixed enzyme on live weight, weight gain, feed conversion ratio, survivability, production number, performance index, production cost of ducks are shown in the Table 2. .Live weight differed significantly among diets and increasing dietary PRP (15-35\%) decreased live weight linearly (Table 2).Ducks on 15\% PRP diet had 4.13-6.15\% increased live weight for supplementation of mixed enzyme depending on age. Respective increased live weight on 25\% PRP diet was 5.04 - 20.01\% for enzyme addition depending on age. Corresponding figures for 35\% PRP diet was 14.36-35.5\%.

Table 2. Growth performance of ducklings on different parboiled rice polish (PRP) diets at different ages

\begin{tabular}{|c|c|c|c|c|c|c|c|c|}
\hline \multirow[t]{2}{*}{ Parameters } & \multirow{2}{*}{$\begin{array}{l}\text { Age } \\
\text { (days) }\end{array}$} & \multicolumn{6}{|c|}{ Diet (D) } & \multirow{2}{*}{$\begin{array}{l}\text { LSD (SED) and leve } \\
\text { of significance + }\end{array}$} \\
\hline & & $D_{1}$ & $D_{2}$ & $\mathrm{D}_{3}$ & $\mathrm{D}_{4}$ & $D_{5}$ & $D_{6}$ & \\
\hline \multirow{13}{*}{$\begin{array}{l}\text { Live weight } \\
\text { (g/duckling) }\end{array}$} & Initial & 45.4 & 45.8 & 45.9 & 45.65 & 45.25 & 45.7 & $0.27^{\mathrm{NS}}$ \\
\hline & 7 & $166.89^{\mathrm{b}}$ & $173.5^{\mathrm{a}}$ & $165.5^{\mathrm{b}}$ & $173.85^{a}$ & $152.85^{\mathrm{C}}$ & $174.8^{\mathrm{a}}$ & $5.672^{\star \star}$ \\
\hline & 14 & $360.3^{\mathrm{bc}}$ & $377.5^{\mathrm{b}}$ & $345.8^{c}$ & $415.0^{a}$ & $320.0^{d}$ & $704.8^{\mathrm{a}}$ & $21.02^{\star \star}$ \\
\hline & 21 & $615.5^{c}$ & $653.35^{\mathrm{bc}}$ & $1616.8^{\mathrm{C}}$ & $724.5^{a}$ & $531.1^{d}$ & $684.5^{\mathrm{b}}$ & $36.67^{\star \star}$ \\
\hline & 28 & $931.75^{\mathrm{C}}$ & $977.55^{b}$ & $921.35^{\mathrm{C}}$ & $1070.9^{a}$ & $782.1^{\mathrm{b}}$ & $1059.45^{a}$ & $25.27^{\star \star}$ \\
\hline & 35 & $1259^{d}$ & $1342^{c}$ & $1259^{d}$ & $1475^{a}$ & $1079^{c}$ & $1444^{\mathrm{b}}$ & $19.23^{\star \star}$ \\
\hline & 42 & $1642^{b}$ & $1737^{\mathrm{C}}$ & $1627^{d}$ & $1865^{a}$ & $1456^{c}$ & $1778^{b}$ & $24.52^{\star \star}$ \\
\hline & 49 & $1895^{d}$ & $1981^{c}$ & $1882^{d}$ & $2159^{a}$ & $1736^{\mathrm{C}}$ & $2086^{\mathrm{b}}$ & $17.78^{\star \star}$ \\
\hline & 56 & $2121^{d}$ & $2231^{c}$ & $2110^{d}$ & $2443^{a}$ & $19782^{c}$ & $2355^{b}$ & $20.74^{\star *}$ \\
\hline & 63 & $2317.75^{d}$ & $2429.5^{c}$ & $2303.5^{d}$ & $2677^{a}$ & $2186.2^{c}$ & $2572.3^{b}$ & $27.29^{\star \star}$ \\
\hline & 70 & $2480.25^{d}$ & $2588^{c}$ & $2459.65^{d}$ & $2878.85^{a}$ & $2359^{c}$ & $2761.05^{b}$ & $24.21^{\star \star}$ \\
\hline & 77 & $2618^{d}$ & $2726^{\mathrm{c}}$ & $2603^{d}$ & $3055^{a}$ & $2505^{c}$ & $2917^{b}$ & $16.62^{\star \star}$ \\
\hline & 84 & $2751^{d}$ & $2883^{c}$ & $2711^{c}$ & $3188^{a}$ & $2580^{f}$ & $3050^{b}$ & $35.38^{\star \star}$ \\
\hline \multirow{12}{*}{$\begin{array}{l}\text { Fed intake } \\
\text { (g/duckling) }\end{array}$} & 7 & $273.7^{b c}$ & $275.8^{\mathrm{abc}}$ & $270.9^{c}$ & $282^{\mathrm{ab}}$ & $249.6^{d}$ & $286^{a}$ & $10.35^{\star *}$ \\
\hline & 14 & $762.3^{\mathrm{ab}}$ & $749.4^{\mathrm{ab}}$ & $688.3^{b}$ & $822.1^{\mathrm{a}}$ & $727.5^{b}$ & $827^{\mathrm{a}}$ & $86.52^{\star}$ \\
\hline & 21 & $1394^{\mathrm{b}}$ & $1434^{\mathrm{b}}$ & $1384^{b}$ & $1692^{a}$ & $1126.5^{c}$ & $1559^{a}$ & $185.70^{* *}$ \\
\hline & 28 & $2473^{b}$ & $2200^{c}$ & $2150^{c}$ & $2332^{\mathrm{bc}}$ & $2100^{c}$ & $2747^{a}$ & $229.70 * *$ \\
\hline & 35 & $3482^{\mathrm{bc}}$ & $3551^{a b}$ & $3396^{\mathrm{bc}}$ & $3965^{a}$ & $3163^{c}$ & $3701^{a b}$ & $426.10^{* *}$ \\
\hline & 42 & $5100^{b}$ & $5210^{\mathrm{ab}}$ & $5003^{b}$ & $5513^{a}$ & $4382^{c}$ & $5490^{a}$ & $299.60^{\star *}$ \\
\hline & 49 & 5965 & 5960 & 5785 & 6528 & 5776 & 6457 & $146.20^{\mathrm{NS}}$ \\
\hline & 56 & $6505.57^{b}$ & $6817.78^{b}$ & $6707.69^{b}$ & $7442.49^{a}$ & $6407.37^{b}$ & $7333.6^{a}$ & $491.80^{\star \star}$ \\
\hline & 63 & 7702.55 & 7663.75 & 7786.55 & $8182.46^{a}$ & 7385.95 & 8047 & $133.38^{\mathrm{NS}}$ \\
\hline & 70 & $7828.25^{b}$ & $8351.38^{\mathrm{ab}}$ & $8267.77^{\mathrm{ab}}$ & $8853.5^{a}$ & $8420.85^{\mathrm{ab}}$ & $8674.65^{a}$ & $580.70^{\star}$ \\
\hline & 77 & $8617^{\mathrm{b}}$ & $8951^{\mathrm{b}}$ & $8847^{\mathrm{a}}$ & $9781^{a}$ & $9813^{\mathrm{a}}$ & $9505^{a}$ & $531.80^{\star *}$ \\
\hline & 84 & 9890.34 & 9704.28 & 10111.75 & 10652.10 & 10416.75 & 10105.98 & $349.800^{\mathrm{NS}}$ \\
\hline
\end{tabular}


Table 2 (contd.) the growth performance of ducklings on different parboiled rice polish (PRP) diets at different ages

\begin{tabular}{|c|c|c|c|c|c|c|c|c|}
\hline \multirow[t]{2}{*}{ Parameters } & \multirow{2}{*}{$\begin{array}{c}\text { Age } \\
\text { (days) }\end{array}$} & \multicolumn{6}{|c|}{ Diet (D) } & \multirow{2}{*}{$\begin{array}{c}\text { LSD (SED) and } \\
\text { level of } \\
\text { significance + }\end{array}$} \\
\hline & & $\mathrm{D}_{1}$ & $\mathrm{D}_{2}$ & $D_{3}$ & $\mathrm{D}_{4}$ & $\mathrm{D}_{5}$ & $\mathrm{D}_{6}$ & \\
\hline \multirow{12}{*}{$\begin{array}{l}\text { Feed } \\
\text { conversion } \\
\text { efficiency }\end{array}$} & 7 & $2.27 a b$ & $2.16 \mathrm{c}$ & $2.26 a b$ & $2.20 \mathrm{bc}$ & $2.30 a$ & $2.22 \mathrm{bc}$ & $0.081^{*}$ \\
\hline & 14 & 2.42 & 2.26 & 2.3 & 2.23 & 2.65 & 2.29 & $0.127^{\mathrm{NS}}$ \\
\hline & 21 & 2.45 & 2.36 & 2.43 & 2.49 & 2.32 & 2.44 & $0.894^{\mathrm{NS}}$ \\
\hline & 28 & $2.79 a$ & $2.36 \mathrm{~b}$ & $2.46 \mathrm{~b}$ & $2.28 \mathrm{~b}$ & $2.85 a$ & $2.71 \mathrm{a}$ & $0.215^{\star \star}$ \\
\hline & 35 & 2.85 & 2.74 & 2.80 & 2.78 & $3.06 a$ & 2.65 & $0.118^{\mathrm{NS}}$ \\
\hline & 42 & 3.20 & 3.08 & 3.17 & 3.03 & 3.11 & 3.15 & $0.055^{\mathrm{NS}}$ \\
\hline & 49 & 3.23 & 3.08 & 3.15 & 3.09 & 3.42 & 3.178 & $0.12^{N S}$ \\
\hline & 56 & 3.14 & 3.12 & 3.60 & 3.121 & 3.33 & 3.18 & $0.198^{\mathrm{NS}}$ \\
\hline & 63 & 3.39ab & $3.22 a b c$ & $3.45 a$ & $3.11 \mathrm{c}$ & $3.45 a$ & $3.19 b c$ & $0.244^{*}$ \\
\hline & 70 & $3.32 b c$ & $3.29 b c$ & $3.43 a b$ & $3.13 c$ & $3.64 a$ & $3.20 b c$ & $0.244^{*}$ \\
\hline & 77 & $3.35 b c$ & $3.34 b c$ & $3.46 b$ & $3.25 c$ & $3.99 a$ & $3.31 b c$ & $0.199 * \star$ \\
\hline & 84 & $3.66 b c$ & $3.42 c$ & $3.80 a b$ & $3.39 c$ & $4.11 a$ & $3.37 c$ & $0.315^{\star \star}$ \\
\hline \multirow{12}{*}{$\begin{array}{l}\text { Performance } \\
\text { index }\end{array}$} & 7 & $7.32 c$ & $8.03 a$ & $7.31 c$ & 7.90ab & $6.59 d$ & $7.87 \mathrm{~b}$ & $0.49 * \star$ \\
\hline & 14 & $14.90 c$ & $16.7 \mathrm{bc}$ & $15.06 \mathrm{c}$ & $18.66 a$ & $12.19 d$ & 17.84ab & $1.827^{\star *}$ \\
\hline & 21 & $25.18 b c$ & $27.68 \mathrm{ab}$ & $25.43 b c$ & $29.14 a$ & $22.92 c$ & $28.06 a b$ & $2.830 \star \star$ \\
\hline & 28 & $33.39 c$ & $41.42 b$ & $37.53 b$ & $47.23 a$ & $27.44 d$ & $39.10 \mathrm{~b}$ & $4.072^{\star \star}$ \\
\hline & 35 & $44.61 \mathrm{c}$ & $49.08 \mathrm{bc}$ & $45.03 c$ & $53.14 a b$ & $35.26 \mathrm{~d}$ & $54.51 a$ & $4.865^{\star \star}$ \\
\hline & 42 & $51.38 \mathrm{c}$ & $56.42 b$ & $51.39 c$ & $61.55 a$ & $46.91 d$ & $56.78 b$ & $2.145^{\star \star}$ \\
\hline & 49 & $58.76 c$ & $64.32 \mathrm{~b}$ & $59.75 c$ & $69.98 a$ & $51.00 d$ & $65.90 \mathrm{ab}$ & $4.529 \star \star$ \\
\hline & 56 & $67.63 \mathrm{~cd}$ & $71.53 \mathrm{bc}$ & $64.93 d$ & $78.68 a$ & $59.34 c$ & $74.21 b$ & $4.372^{\star \star}$ \\
\hline & 63 & $68.44 d$ & $75.56 c$ & $66.90 \mathrm{~d}$ & $86.07 a$ & $63.42 d$ & $80.76 b$ & 5.156 *夫 \\
\hline & 70 & $77.15 c$ & $78.79 c$ & $71.84 d$ & $92.13 a$ & $64.88 c$ & $86.41 b$ & $4.728^{\star \star}$ \\
\hline & 77 & $78.16 \mathrm{~cd}$ & $81.63 c$ & $75.25 d$ & $94.04 a$ & $62.75 c$ & $88.16 \mathrm{~b}$ & 4.996 ** \\
\hline & 84 & $75.38 c$ & $84.33 b$ & 71.25 & $94.04 a$ & $62.79 d$ & $90.74 a b$ & $6.604^{\star \star}$ \\
\hline Livability \% & 84 & 100 & 100 & 80.00 & 90.00 & 90.00 & 100 & $5.317^{\mathrm{NS}}$ \\
\hline \multicolumn{9}{|c|}{ Cost of production duck } \\
\hline \multicolumn{2}{|c|}{ Feed cost/duck } & 105.30 & 107.97 & 112.18 & 111.97 & 111.97 & 117.71 & $4.096^{\mathrm{NS}}$ \\
\hline \multicolumn{2}{|c|}{ Feed cost/kg duck } & $38.18 b c$ & $37.46 \mathrm{bc}$ & $41.38 a b$ & $4 . .36 a$ & $43.36 \mathrm{a}$ & $38.59 b c$ & $3.773^{\star}$ \\
\hline \multicolumn{2}{|c|}{ Total cost (Tk. / duck) } & 126.83 & 129.74 & 133.98 & 133.77 & 133.77 & 139.51 & $2.192^{\text {NS }}$ \\
\hline \multicolumn{2}{|c|}{ Total cost (Tk./ kg duck) } & $46.10 \mathrm{bc}$ & $45.02 \mathrm{c}$ & 49.42ab & $51.86 a$ & $51.86 a$ & $45.74 \mathrm{bc}$ & $3.840^{\star \star}$ \\
\hline \multicolumn{2}{|c|}{ Profit (Tk/kg duck) } & 13.90ab & $14.99 a$ & $10.58 \mathrm{bc}$ & $8.15 c$ & $8.15 c$ & $14.26 a b$ & $3.840^{\star \star}$ \\
\hline
\end{tabular}

$+\mathrm{NS}, \mathrm{P}>0.05 ;{ }^{*}, \mathrm{P}<0.05 ; * * \mathrm{P}<0.01 ;$ All SED are against $5 \mathrm{df}$

$D_{1}=15 \%$ PRP diet; $D_{2}=D_{1}+$ mixed enzyme; $D_{3}=25 \%$ PRP diet; $D_{4}=D_{3}+$ mixed enzyme; $D_{5}=35 \% P R P$ diet; and $D_{6}$

$=D_{5}+$ mixed enzyme

Feed intake differed significantly among diets and feed intake almost decreased linearly with increasing PRP levels (Table 2) but increased with supplementation of mixed enzyme. At 15\% PRP diet had 0.77$6.70 \%$ increased feed intake for addition of mixed enzyme, except at 14, 28, 49, 63 and 84 days where feed intake decreased for enzyme supplementation. At 25\% PRP diet increased feed intake, was 4.09$22.25 \%$ for enzyme supplementation. Corresponding increase for 35\% PRP diet was $3.02-38.39 \%$, except $77 \& 84$ days where feed intake decrease for enzyme supplementation.

Increasing PRP level in diet decreased feed conversion efficiency (FCE) linearly at all ages (Table 2). Addition of mixed enzyme had a significant $(P<0.01)$ effect on FCE at different dietary PRP levels. A depressing feed conversion on increasing proportion of PRP in diet found. This may imply that due to higher phytin $\mathrm{P}$ concentration and NSP decline nutrients utilization with a consequent reduced poorer FC on PRP diets. 
Performance index $(\mathrm{PI})$ differed significant $(\mathrm{P}<0.01)$ among diets. At 15,25 , and $35 \% \mathrm{PRP}$, increased $\mathrm{PI}$ were 2.12- $24.04,8,07-28.65$ and $19.40-54.59 \%$ respectively for enzyme supplementation. Livability did not differ significantly among 6 different diets. Total cost of production and profit on different dietary treatments are increasing dietary PRP and with addition of mixed enzyme increased profit. Feed cost $/ \mathrm{kg}$ duck was decreased significantly on three enzymatic diets than without supplementation of enzyme in PRP diets. At 15, 25, and 35\% PRP levels increased profit for enzyme supplementation in diet were 7.84, 57.65 and $74.96 \%$ respectively.

Here is significant $(P<0.01)$ effect $t$ for mixed enzyme supplementation on the performance parameter of dressing yield and meat yield characteristics with increasing PRP levels (Table 3). For addition of enzyme gave improve response of total meat, dark meat, breast meat, drumstick meat and dressing percentage.

Table 3. Dressing yield characteristics of ducks on different PRP diet without and with mixed enzyme supplementation

\begin{tabular}{|c|c|c|c|c|c|c|c|c|c|c|c|}
\hline \multirow[t]{2}{*}{ Parameters } & \multirow[t]{2}{*}{ Sex } & \multicolumn{6}{|c|}{ Treatments } & \multirow[t]{2}{*}{ Mean } & \multicolumn{3}{|c|}{ LSD (SED) and level of significance + } \\
\hline & & $D_{1}$ & $\mathrm{D}_{2}$ & $\mathrm{D}_{3}$ & $\mathrm{D}_{4}$ & $D_{5}$ & $D_{6}$ & & $\mathrm{D}$ & $\mathrm{S}$ & $\mathrm{D} \times \mathrm{S}$ \\
\hline \multirow[t]{3}{*}{ Dressing yield \% } & $\mathrm{M}$ & 60.60 & 61.29 & 60.40 & 63.02 & 58.85 & 62.47 & 60.51 & \multirow[t]{3}{*}{$0.514^{* *}$} & \multirow[t]{3}{*}{$0.134^{\star *}$} & \multirow[t]{3}{*}{$0.637^{\mathrm{NS}}$} \\
\hline & $\mathrm{F}$ & 59.56 & 60.29 & 59.28 & 62.02 & 57.74 & 61.50 & 60.66 & & & \\
\hline & Mean & 60.09 & 60.79 & 59.84 & 62.52 & 58.29 & 61.98 & 60.58 & & & \\
\hline \multirow[t]{3}{*}{ Total meat \% } & $\mathrm{M}$ & 30.04 & 31.93 & 29.05 & 73.64 & 28.54 & 32.99 & 30.34 & \multirow[t]{3}{*}{$0.403^{* *}$} & \multirow[t]{3}{*}{$0.011^{* *}$} & \multirow[t]{3}{*}{$0.796^{\mathrm{NS}}$} \\
\hline & $\mathrm{F}$ & 28.34 & 30.61 & 27.69 & 32.50 & 27.37 & 32.08 & 30.46 & & & \\
\hline & Mean & 29.19 & 31.27 & 28.37 & 33.05 & 27.95 & 32.53 & 30.39 & & & \\
\hline \multirow[t]{3}{*}{ Dark meat \% } & $\mathrm{M}$ & 16.11 & 16.85 & 16.09 & 18.25 & 15.54 & 17.54 & 16.34 & \multirow[t]{3}{*}{$0.295^{\star *}$} & \multirow[t]{3}{*}{$0.077^{* \star}$} & \multirow[t]{3}{*}{$0.418^{\mathrm{NS}}$} \\
\hline & $\mathrm{F}$ & 15.49 & 16.30 & 15.37 & 17.85 & 15.25 & 17.11 & 16.62 & & & \\
\hline & Mean & 15.80 & 16.57 & 15.73 & 18.05 & 15.39 & 17.32 & 16.49 & & & \\
\hline \multirow[t]{3}{*}{ Breast meat \% } & $\mathrm{M}$ & 14.50 & 14.12 & 13.87 & 16.01 & 13.21 & 15.81 & 14.43 & \multirow[t]{3}{*}{$0.241^{\star \star}$} & \multirow[t]{3}{*}{$0.063^{\star \star}$} & \multirow[t]{3}{*}{$0.456^{\mathrm{NS}}$} \\
\hline & $\mathrm{F}$ & 14.01 & 14.32 & 13.02 & 15.74 & 12.88 & 15.50 & 14.52 & & & \\
\hline & Mean & 14.26 & 14.57 & 13.44 & 15.87 & 13.04 & 15.66 & 14.57 & & & \\
\hline \multirow[t]{3}{*}{ Thigh meat \% } & $\mathrm{M}$ & 6.19 & 6.47 & 6.18 & 7.04 & 6.11 & 6.70 & 6.31 & \multirow[t]{3}{*}{$0.085^{\star \star}$} & \multirow[t]{3}{*}{$0.022^{\star *}$} & \multirow[t]{3}{*}{$0.132^{\mathrm{NS}}$} \\
\hline & $\mathrm{F}$ & 6.11 & 6.31 & 5.99 & 6.70 & 5.99 & 6.49 & 6.42 & & & \\
\hline & Mean & 6.15 & 6.38 & 6.06 & 6.87 & 6.05 & 6.59 & 6.36 & & & \\
\hline \multirow{3}{*}{$\begin{array}{l}\text { Drumstick meat } \\
\%\end{array}$} & $\mathrm{M}$ & 6.12 & 6.24 & 6.18 & 6.80 & 6.00 & 6.71 & 6.16 & \multirow[t]{3}{*}{$0.069^{* *}$} & \multirow[t]{3}{*}{$0.018^{*}$} & \multirow[t]{3}{*}{$0.098^{\star \star}$} \\
\hline & $\mathrm{F}$ & 5.97 & 6.09 & 6.10 & 6.66 & 5.83 & 5.55 & 6.22 & & & \\
\hline & Mean & 6.04 & 6.17 & 6.14 & 6.73 & 5.92 & 6.13 & 6.19 & & & \\
\hline Abdominal fat \% & $\mathrm{M}$ & 0.81 & 1.17 & 0.92 & 0.99 & 0.91 & 0.94 & 0.62 & $0.038^{\mathrm{NS}}$ & $0.039^{\star \star}$ & $0.132^{\text {NS }}$ \\
\hline & $\mathrm{F}$ & 0.33 & 0.36 & 0.34 & 0.38 & 0.38 & 0.36 & 0.69 & & & \\
\hline & Mean & 0.57 & 0.77 & 0.63 & 0.68 & 0.64 & 0.65 & 0.66 & & & \\
\hline Blood loss \% & $\mathrm{M}$ & 4.68 & 5.04 & 4.79 & 5.25 & 4.49 & 5.21 & 4.61 & $0.156^{\star \star}$ & $0.040^{\star \star}$ & $0.158^{\text {NS }}$ \\
\hline & $\mathrm{F}$ & 4.21 & 4.40 & 4.14 & 4.77 & 4.14 & 4.68 & 4.69 & & & \\
\hline & Mean & 4.44 & 4.72 & 4.47 & 5.00 & 4.32 & 4.94 & 4.65 & & & \\
\hline Feather loss \% & $\mathrm{M}$ & 6.76 & 6.93 & 6.67 & 7.76 & 6.64 & 7.00 & 6.67 & $0.139^{\star *}$ & $0.037^{\star *}$ & $0.197^{\star \star}$ \\
\hline & $\mathrm{F}$ & 6.19 & 6.53 & 6.35 & 7.09 & 6.33 & 6.83 & 6.85 & & & \\
\hline & Mean & 6.47 & 6.73 & 6.51 & 7.43 & 6.45 & 6.92 & 6.76 & & & \\
\hline
\end{tabular}

+NS, P>0.05; * $\mathrm{P}<0.05 ;{ }^{* *} \mathrm{P}<0.01 ;$ All SED are against $5 \mathrm{df}$

$D_{1}=15 \%$ PRP diet; $D_{2}=D_{1}+$ mixed enzyme; $D_{3}=25 \%$ PRP diet; $D_{4}=D_{3}+$ mixed enzyme; $D_{5}=35 \% P R P$ diet; and $D_{6}=D_{5}+$ mixed enzyme

Decreasing live weight with increasing concentration of dietary PRP coincide with some previous findings (Islam et al 1996; Azam and Howlider, 1998; Sayre et al. 1987; Scholtyseek et al 1986). Improved growth of broiler on increasing dietary PRP following addition of mixed enzyme agreed with Moshad (2001).

Decrease feed intake on increasing dietary PRP agreed with the report of Sayre et al. (1987), Mahbub (1989), Karim (1983). Feed intake was improved for addition of mixed enzyme and rate of improvement was higher at increasing PRP level. Such result is supported by previous findings (Ravindran et al 1995, Zyla et al 1999, Gippert et al 1999, Moshad. 2001).

Increasing PRP level in diet decreased feed conversion efficiency (FCE) linearly at all ages .This result agreed with the investigation of Warren and Farrell, (1990), Kanaya et al (1976). Improved FCE on increasing PRP levels in diet with addition of mixed enzyme agreed with Moshad (2001), Ravindran et al (1995). 
At 15, 25, and 35\% PRP levels increased profit for enzyme supplementation in diet were 7.84, 57.65 and $74.96 \%$ respectively. Such results obtained coincide with the findings of several authors (Farrell et al 1993; Kies et al 2001; QuMingren et al. 1999 and Mikulshi et al. 1999).

Moshad (2001), Preston et al. (2000), Jamroz et al. (1996) and Ferguson et al. (1998) have reported increased carcass yield for addition of enzymes. Dressed weight was a function of live weight. A positive correlation of dressed weight with live weight or age obtained coincide with the findings of Mcnally and Spicknall (1949), Jap et al. (1950) incase of broiler.

As increasing use of dietary PRP up to $35 \%$ in diet with mixed enzyme ( Phytase $1000 \mathrm{FYT} / \mathrm{kg}$ and carbohydrase $120 \mathrm{ppm} / \mathrm{kg}$ ) gave best performance of duck meat production, it may be worthwhile to further investigate whether a higher level above $35 \%$ might produce improve or similar growth leading to a further increase in profitability.

\section{References}

Azam, G. and Howlider, M.A.R. 1998. Use of autoclave parboiled rice polish as substitute of grain in broiler diet. Journal of Applied Animal Research 14:181-186.

Asian Livestock, 1978. FAO. Regional Animal Production and Health Commision for Asia and the Pacific (APHCA). 10(4):6.

Banerjee, G.C. 1992. Poultry, $3^{\text {rd }}$ Edn. Oxford and IBH Publishing Co Pvt. Ltd. New Delhi, 105-108.

Barber, S.K. and Barber, P.K. 1980. Utilization of rice bran by broiler and layer chicken. Poultry Science, 59(8):P $1012-1017$.

Eshawaraiah, Reddy, C.V. and Rao, P.V. 1988. Effect of autoclaving and solid substrate fermentation of raw, deoiled and parboiled rice polishing in broiler diets. Indian Journal of Animal Science. 58:377-381.

Farrel, D.J. and martin. E. 1993. Feed enzymes in Poultry Nutrition: recent finding. Recent advances in Animal Nutrition in Australia, pp. 166- 176.

Ferguson,N.S., Gates, R.S., Taraba, J.L., Cantor, A.H., Pescatore, A.J., Straw,M.L., Ford, M.J. and Burnham, D.J. 1998. The effect of dietary protein and phosphorus on ammonia concentration and litter composition in broilers. Poultry Science, 77:8, 1085-1093; 37.

Gippert, T., Kis, I., Gerendai, D., El- Sherif, K. Hullar, I. 1999. The effect of phytase enzyme in broiler nutrition. Allattenyes-esTakarmanyozas. In : Balayar, G.T. 1996. Journal of Animal Science, 16: 755-760.

Islam, M.N., Islam.M.S., Hashem, M.A., and Howlider, M.A.R. 1996. The effect of parboiled rice polish diet on growth and meat yield of broilers. Progressive Agriculture, 7: 87-91.

Jaap,R.G.; Renard, M.M. and Buckingham, R.D. 1950. Dressed eviscerated meat yields from chickens at twelve weeks of age. Poultry Science, 29:874-880.

Karim, R.M.M. 1983. Study on the effect of using various levels of rice polish in growing chick rations. M. Sc. Thesis, Department of Poultry Science, Bangladesh Agricultural University, Mymensingh.

Mahbub, A.S.M., Rahman, M.A. and Reza, A. 1989. Use of rice polish as partial replacement of wheat in the diet of growing chicks. Bangladesh Journal of Animal Science, 18: 99-104.

Moshad, M.A. 2001. Use of phytase and carbohydrase enzyme for better utilization of parboiled rice polish based diet in broilers. M.S. Thesis, Department of Poultry Science, Bangladesh Agricultural University, Mymensingh.

Preston, C.M., McCracken,K.J. and McAllister,A. 2000. Effect of diet form and enzyme supplementation on growth efficiency and energy utilization of wheat based diets for broilers. British Poultry Science, 41:324-331.

Ravindran, V., Bryden, W. L. and Kornegay, E. T. 1995. Phytase : occurance, bioavailability and implications in poultry nutrition. Poultry and Avian Biology Reviews, 6:125-143.

Sayre, R.N., Earl, N., Kratzer, F.H. and Saunders, R.M. 1987. Nutritional qualities of stabilized and raw rice bran for chicks. Poultry Science, 66: 493-499.

Scholtyseek, S.; Landfried, K.E. and Swierczewska, E. 1986. Improvement of broiler rations with domestic polish feed components. Poultry Abstracts, Vol. 12, No. 11.

Zyla, K., Gogol, D., Koreleski,J., Swiatkiewicz,S. and Ledoux, D.R.1999. Simultaneous application of phytase and Xylanse to broiler feeds based on wheat: feeding experiment with growing broiler. Journal of the science of food and agriculture, 79: 18411848. 